\title{
Diversidade e usos de plantas medicinais nos quintais da comunidade de São João da Várzea em Mossoró, RN
}

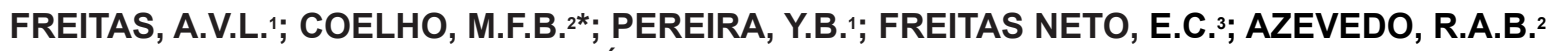
1Universidade Federal Rural do Semi-Árido-UFERSA, Programa de Pós-Graduação em Fitotecnia, Km 47 da BR110, Caixa Postal 137, Mossoró - RN, CEP 59625-900 2Universidade da Integração Internacional da Lusofonia Afro Brasileira-UNILAB, Instituto de Desenvolvimento Rural, Av. da Abolição, 7. Redenção-CE, CEP 62790-000. ${ }^{3}$ Universidade do Estado do Rio Grande do Norte-UERN, Avenida Profo Antônio Campos, S/N. Bairro: Costa e Silva. CEP: 59.625-620 *Autor para correspondência: coelhomfstrela@gmail.com

RESUMO: Nos quintais do nordeste brasileiro são mantidas tradicionalmente diversas espécies vegetais para o tratamento de doenças. O objetivo do presente trabalho foi realizar um levantamento das espécies medicinais em quintais na Comunidade de São João da Várzea, Mossoró, Rio Grande do Norte, Brasil. Participaram do estudo 22 informantes e foram aplicadas para a coleta de dados técnicas de observação direta e participante, turnê guiada, lista livre e entrevistas semiestruturadas. Verificou-se a presença de 57 espécies pertencentes a 37 famílias botânicas. As espécies com maior frequência de ocorrência foram goiabeira ( $P$ sidium guajava L.) e coqueiro (Cocos nucifera L.), presentes em 59\% e 55\% dos quintais, respectivamente. Para estas espécies houve maior índice de concordância de uso (CUPc de 61,5\% e 92,3\%). Foram citadas 67 patologias e o maior número de plantas medicinais foi indicado para o tratamento de gripe, disenteria, inflamações e como cicatrizante de ferimentos. O principal modo de administração foi por via oral, na forma de decocção (58\%) e as folhas foram as partes mais usadas (32,6\%). Os quintais são sistemas que atuam como uma estratégia de uso mais sustentável dos recursos medicinais por conservarem também as espécies nativas. As espécies medicinais mais frequentes nos quintais são arvores frutífero-alimentícias importantes para a renda familiar. A gripe e desinteria são os principais agravos de saúde tratados com espécies medicinais pela comunidade. Ziziphus joazeiro Mart., Citrus X limonia (L.) Osbeck, Punica granatum L., Cocos nucifera L. e Psidium guajava L. são espécies que merecem maiores estudos farmacológicos.

Palavras-chave: Etnobotânica, medicina tradicional, medicina caseira

\begin{abstract}
Diversity and uses of medicinal plants in homegardens at the community São João da Varzea, Mossoró, RN. Traditionally, at homegardens in the northeastern of Brazil, several plant species are employed for the treatment of diseases. The aim of this study was to research the species of medicinal use in homegardens of São João da Varzea, Mossoró, Rio Grande do Norte, Brazil. 22 homegardens were studied through the techniques of direct and participant observations, guided tours, free list and semi structured interviews. We verified the presence of 57 botanical species belonging to 37 families. The two species with the highest frequency of occurrence were the guava (Psidium guajava L.) and the coconut (Cocos nucifera L.), present in $59 \%$ and $55 \%$ of the homegardens, respectively. For these species there was more agreement of use (CUPc $61.5 \%$ and 92.3\%). 67 diseases, including influenza and dysentery were the most common ones mentioned during the interviews. The main mode of administration was orally, in the form of decoction (58\%), and the leaves were the most employed component (32.6\%). Homegardens are systems that act as a more sustainable use of medicinal resources strategy since they conserve native species. The most common medicinal plants in the homegardens are the fruitful-food trees, important to the family income. The flu and the dysentery are the main health problems treated with medicinal plants by the community. The Ziziphus joazeiro Mart., Citrus $X$ limonia (L.) Osbeck, Punica granatum L., Cocos nucifera L. and Psidium guajava L. are species that deserve further pharmacological studies.
\end{abstract}

Keywords: Etnobotany, traditional medicine, folk medicine.

Recebido para publicação em 22/07/2014

Aceito para publicação em 13/01/2015

10.1590/1983-084X/14_080

Rev. Bras. PI. Med., Campinas, v.17, n.4, supl. II, p.845-856, 2015. 


\section{INTRODUÇÃO}

Os quintais podem ser definidos como uma área de produção localizada perto da casa, onde são cultivadas espécies agrícolas e florestais, e são criados pequenos animais domésticos (Kumar \& Nair, 2004). A importância dos quintais agroflorestais é relatada por vários autores, principalmente quanto à segurança alimentar e nutricional, aumento da renda familiar, introdução e domesticação de espécies, eficiência do uso da terra resultante da diversidade biológica e conservação de espécies (Angel-Perez \& Martin, 2004; Moura \& Andrade, 2007; Leonel, 2010).

Cada indivíduo percebe e utiliza os recursos presentes nos espaços que maneja de maneira distinta, acumulando conhecimento ao longo do tempo e o repassando para gerações futuras. Nesse sentido, as plantas são utilizadas para múltiplas finalidades, destacando-se, em vários estudos realizados em quintais, o uso de plantas alimentícias e medicinais (Albuquerque et al., 2005; Florentino et al., 2007; Moura \& Andrade, 2007; Pasa et al., 2008; Guarim Neto \& Amaral, 2010; Morais, 2011; Freitas et al., 2012; Siviero et al., 2012; Liporacci \& Simão, 2013).

O homem utiliza as plantas como alternativa terapêutica na perpetuação de informações valiosas, muitas vezes próprias de sua cultura. Este fator faz com que cada sociedade ou comunidade possua seu próprio sistema de classificação, crenças e métodos populares capazes de promover a cura dos seus próprios males (Freitas et al., 2012).

No entanto, observa-se uma tendência à redução e ao desaparecimento dos conhecimentos tradicionais motivados pela ação constante do processo de modernização (Diegues, 2008). Desta forma, o resgate desses conhecimentos tem merecido atenção especial nos últimos anos e a valorização e resgate de conhecimentos e recursos genéticos tradicionais são também processos de manutenção da diversidade (Delwing et al., 2007).

Uma das grandes contribuições dos quintais no nordeste do Brasil é o fornecimento de plantas para uso medicinal. Por exemplo, Aguiar \& Barros (2012), conduziram um levantamento de plantas medicinais em 21 quintais de Demerval Lobão, Piauí, e verificaram a presença de 100 espécies vegetais. No Estado do Rio Grande do Norte, dezessete trabalhos etnobotânicos foram realizados, sendo que a maioria investigou o uso de plantas medicinais nas comunidades (Guerra et al., 2007; Mosca \& Loiola, 2009; Guerra et al., 2010; Roque et al., 2010; Silva \& Freire, 2010; Freitas et al., 2012; Paulino et al., 2012).

O objetivo do presente trabalho foi realizar um levantamento etnobotânico em quintais da comunidade de São João da Várzea em Mossoró $\mathrm{RN}$, enfatizando o uso de plantas medicinais.

\section{MATERIAL E MÉTODO}

A comunidade São João da Várzea localizase nas coordenadas $5^{\circ} 18^{\prime} 19,5^{\prime \prime} \mathrm{S}$ e $037^{\circ} 24^{\prime} 46,8^{\prime \prime} \mathrm{W}$, altitude de $28 \mathrm{~m}$, distante $18 \mathrm{~km}$ da sede do município, Mossoró. O município possui clima do tipo quente e seco, com precipitação pluviométrica média anual de $695,8 \mathrm{~mm}$; período chuvoso entre os meses de fevereiro a abril; temperatura média anual em torno de $27,2^{\circ} \mathrm{C}$; umidade relativa média anual de 70\%; e 2700 horas de insolação (IDEMA, 2008).

A comunidade São João da Várzea está inserida no Polo Passagem do Rio que é composto por cerca de 400 famílias distribuídas em onze comunidades e dois Assentamentos do Programa Nacional de Crédito Fundiário (EMATER, 2012).

Inicialmente, foram realizadas duas oficinas com os moradores da comunidade, nas quais foi apresentada e discutida a proposta de trabalho, enfatizando os objetivos, a importância dos mesmos e a metodologia a ser adotada. Além disso, foi esclarecido que a participação dos informantes na pesquisa estava condicionada à sua aceitação, podendo os mesmos desistir a qualquer momento sem qualquer prejuízo. Nessas oficinas foi elaborado o Termo de Anuência Prévia conforme orienta o Conselho de Gestão do Patrimônio Genético (Azevedo, 2005).

Foram selecionados com o uso da técnica de amostragem "Bola de neve" (Bailey, 1994) 22 informantes. Utilizou-se como critério para a seleção dos informantes, além de aceitar participar da pesquisa, ser o responsável pela manutenção do quintal e conhecer bem as espécies nele presentes. A partir do contato inicial com a comunidade, um primeiro informante foi reconhecido, que passou a indicar outro e assim, sucessivamente, até envolver todos os informantes da comunidade.

Foram realizadas de duas a cinco visitas por quintal no período de junho a dezembro de 2012, sendo utilizadas diferentes técnicas de coleta de dados. O levantamento etnobotânico foi realizado nos espaços domésticos com o uso de técnicas de observação direta e participante, entrevistas semiestruturadas e listagem livre (Albuquerque et al., 2010).

Os informantes foram indagados sobre 0 uso e manejo das plantas e as partes utilizadas para cada uso, abordando-se questões específicas sobre cada espécie presente nos espaços domésticos. Nesse caso, as entrevistas foram feitas durante a verificação in loco para cada espécie citada por cada informante, utilizando-se o método turnê guiada 
ou "walk-in-the-woods" que consiste em ir até o campo com o informante, visando fundamentar e validar os nomes das plantas citadas nas entrevistas (Albuquerque et al., 2010).

Durante a turnê-guiada, algumas espécies foram herborizadas de acordo com técnicas estabelecidas em coletas botânicas, para posterior identificação taxonômica por especialistas do Herbário da Universidade Federal Rural do Semi Árido. A nomenclatura das espécies e seus autores foram confirmados utilizando-se a base de dados Trópicos $^{\circledR}$ do Missouri Botanical Garden (MOBOT, 2014). O sistema de classificação de plantas utilizado foi o APG II (APG, 2003). À medida que foram coletadas, as informações foram compiladas sob a forma de um banco de dados, utilizando-se matrizes de textos (Amorozo \& Viertler, 2010).

O Índice de Concordância de Uso (CUPc) foi calculado de acordo com Amorozo \& Gely (1988) e o Índice de Shannon-Wiener foi calculado conforme sugerido por Begossi (1996).

\section{RESULTADOS E DISCUSSÃO}

$\mathrm{Na}$ comunidade São João da Várzea residiam 53 famílias e a pesquisa abrangeu um total de 22 informantes de diferentes famílias, o que correspondeu a $42 \%$ dos quintais. Deste total de informantes, 15 (68\%) eram do sexo feminino e $7(32 \%)$ do sexo masculino. Considerando-se o critério de seleção da entrevista, verificou-se a predominância da mulher nas atividades realizadas nos quintais pois os homens lidam com atividades externas aos arredores das residências, sejam estas remuneradas ou não. Em outros trabalhos sobre quintais, as mulheres também corresponderam à maior parcela dos entrevistados (Amorozo, 2002; Florentino et al., 2007; Moura \& Andrade, 2007; Freitas et al., 2012).

A idade dos informantes variou de 23 a 73 anos, com média de 50 anos, sendo que $45 \%$ destes apresentam idades inferiores a 50 anos e $55 \%$, idades superiores. Faixa etária semelhante foi encontrada em Caruaru-PE por Florentino et al. (2007); em Mirassol do Oeste-MT, por Carniello et al. (2010) e em São Miguel-RN, por Freitas et al. (2012). Os informantes residiam na comunidade há um tempo médio de 28 anos, variando de 1 a 65 anos. Do total de informantes, 7 (32\%) afirmaram que nasceram e sempre residiram na comunidade, tendo estes de 47 a 65 anos de idade. A comunidade São João da Várzea é uma das comunidades mais antigas de Mossoró, sempre se destacando como comunidade polo.

Dos 22 informantes, 14 (64\%) eram naturais de Mossoró-RN e apenas um era natural de outro estado, a Paraíba. Os demais informantes eram de outros municípios do Rio Grande do Norte, tais como: Alexandria, Almino Afonso, Caicó, Frutuoso Gomes, Macau, Marcelino Vieira e Martins. O local de nascimento e crescimento do informante pode implicar diretamente no seu conhecimento acerca dos recursos vegetais e animais de determinada região, de vez que o sistema de classificação, o uso, o manejo, a nomenclatura e outros fatores variam de acordo com a cultura e o ambiente do local de origem e do atual.

A grande maioria, 18 informantes (82\%), nasceu e viveu a infância e adolescência na zona rural, ao passo que apenas $4(18 \%)$ nasceram na zona urbana. Esta informação é relevante na medida em que na zona rural as crianças e adolescentes possuem maior contato e vivência com os recursos da natureza e suas interações com o ambiente, o que resulta em maior acúmulo de conhecimento sobre esses recursos, seus usos e manejos. De acordo com Amorozo (2002), o cultivo em comunidades agrícolas tradicionais é algo que se aprende muito cedo, na medida em que as crianças acompanhando os adultos às roças.

Quanto ao estado civil, 17 (77\%) eram casados, $3(14 \%)$ eram divorciados, $1(4,5 \%)$ era viúvo e $1(4,5 \%)$ era solteiro. Segundo Freitas et al. (2012), a condição casado poderá estar relacionada ao maior conhecimento sobre as plantas, sobretudo as medicinais, pois geralmente a existência dos filhos implica na busca de soluções práticas e imediatas para o tratamento de doenças. Apesar de um dos informantes possuir nível superior completo em Agronomia, os demais apresentam baixa escolaridade, tendo 15 (68\%) deles cursado apenas as séries iniciais do nível fundamental. A baixa escolaridade também foi encontrada em outros estudos (Amaral \& Guarim Neto, 2008; Carniello et al., 2010; Freitas et al., 2012; Morais, 2011).

Todos os informantes desenvolviam atividades agropecuárias, sendo que $20(91 \%)$ deles dependiam financeiramente dessas atividades, seja exclusivamente ou de forma associada a outras atividades não agrícolas, como costura ou empregos em empresas de cerâmica. Foram identificadas três formas de posse da terra, onde 10 (45\%) são proprietários, 9 (41\%) são parceiros e 3 (14\%) são posseiros. Vale ressaltar que o sistema de parceria adotado é informal, sendo firmado verbalmente acordo de recompensa pelo uso da terra. Na maioria dos casos, esse acordo acontece por meio do sistema denominado de "terça", no qual um terço da produção é repassado ao proprietário da terra como forma de pagamento de seu uso.

Todas as famílias possuiam algum tipo de benefício, seja aposentadoria ou de programa de transferência direta de renda, como o Programa Bolsa Família do Ministério do Desenvolvimento

Rev. Bras. PI. Med., Campinas, v.17, n.4, supl. II, p.845-856, 2015. 
Social e Combate à Fome (MDS). Resultados semelhantes foram encontrados por Visbiski (2003) em Imaruí-SC, que mostrou a forte participação da aposentadoria como principal fonte geradora de renda para $27 \%$ dos entrevistados. No Rio Grande do Norte, Freitas (2009) e Morais (2011) relataram semelhante composição da renda familiar.

Nos quintais estudados foram citadas 57 espécies com propriedades medicinais pertencentes a 37 famílias botânicas e a família Lamiaceae apresentou o maior número de espécies (Tabela 1).

A maior representatividade da família Lamiaceae pode ser explicada por sua distribuição cosmopolita e sua grande importância terapêutica, comprovada em estudos anteriores (Mosca \& Loiola, 2009; Freitas et al., 2012; Paulino et al., 2012; Alves \& Povh, 2013). As espécies desta família se destacam por ser ricas em óleos essenciais, o que confere a estas espécies propriedades aromáticas e medicinais (Abdel-Mogib et al., 2002).

Outras famílias botânicas que também se destacaram no estudo foram Apocynaceae (3 spp.), Euphorbiaceae (3 spp.), Fabacea- Caesalpinioideae (3 spp.), Amaryllidaceae (2 spp.), Anacardiaceae (2 spp.), Crassulaceae (2 spp.), Cucurbitaceae (2 spp.), Rubiaceae (2 spp.) e Rutaceae (2 spp.). As demais famílias identificadas foram representadas por apenas uma espécie cada.

As espécies mais frequentes nos quintais encontram-se na Figura 1. Ziziphus joazeiro, Malpighia glabra, Citrus X limonia, Cocus nucifera e Psidium guajava estavam em mais de $30 \%$ dos quintais. Estas espécies são usadas como medicinais, mas também são consideradas frutíferas/alimentícias e importantes no aumento da renda familiar através da venda de castanha ( $C$. nucifera) e de doces e compotas (C. nucifera, Citrus $X$ limonia e $P$. guajava).

Esses resultados corroboram com os encontrados por outros autores que constataram que as espécies mais frequentes nos quintais são as frutíferas, observando-se predominância de arvores (Pasa et al., 2005; Florentino et al., 2007). A preferência pelo cultivo de plantas arbóreas segue um padrão para todos os quintais, sendo justificada pela resistência destas espécies às adversidades climáticas, já que são mantidas sem muitas exigências de manejo (Florentino et al., 2007). Espécies arbóreas, além de favorecer o estabelecimento de um microclima favorável, podem beneficiar o desenvolvimento de outras espécies (Wezel \& Bender, 2003; Kumar \& Nair, 2004).

As espécies Cocos nucifera, Croton campestris e Scoparia dulcis apresentaram mais de setenta indivíduos por quintal (Figura 2). Croton campestris é nativa, frequente na caatinga e apresenta diferentes usos como medicinal (Alves et al., 2012) enquanto Scoparia dulcis é ruderal e presente em todo o Brasil.

Estudos conduzidos no Sul do país mostraram outro padrão de frequência de espécies medicinais nesses espaços domésticos. Rondon Neto et al. (2004) estudando quintais agroflorestais no município de Teixeira Soares-PR, verificaram que as espécies de maior frequência foram Cymbopogon citratus Stapf e Pneumus boldus Molina. Em Imaruí, Santa Catarina, Costantin (2005) observou, na maioria dos quintais, que as plantas medicinais apresentaram um papel de destaque, sendo a hortelã (Mentha spp.), o boldo (Vernonia spp.) e o

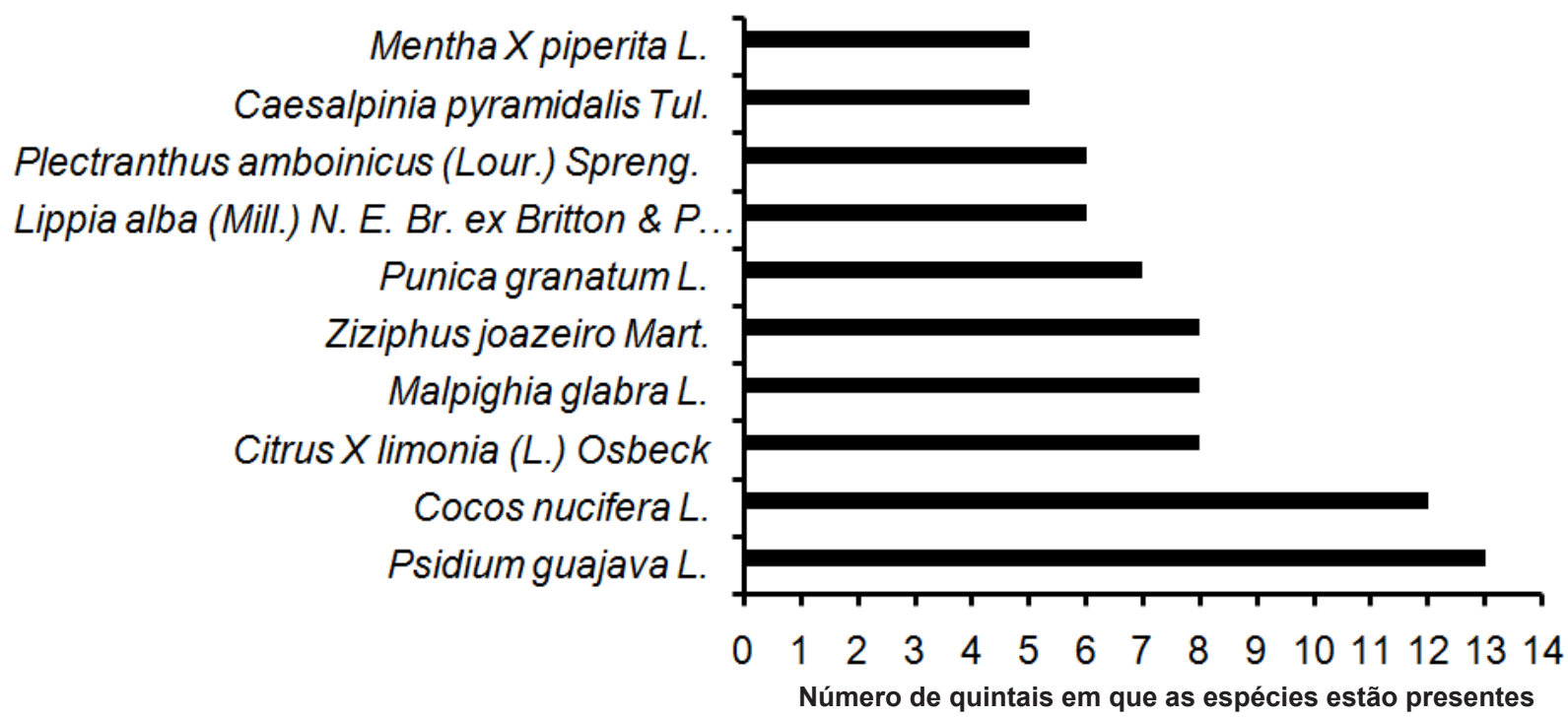

FIGURA 1. Espécies medicinais e número de quintais em que estão presentes na Comunidade de São João da Várzea, Mossoró, RN. 2012

Rev. Bras. PI. Med., Campinas, v.17, n.4, supl. II, p.845-856, 2015. 
TABELA 1. Espécies presentes em espaços domésticos citadas para fins medicinais por informantes de São João da Várzea, Mossoró-RN. Origem ( $E=$ exótica; $N=$ nativa), Parte utilizada ( $A=$ Bulbo; $B=C a s c a ; C=$ Caule; $\mathrm{D}=\mathrm{Flor}$; $\mathrm{E}=$ Folha; $\mathrm{F}=$ Fruto; $\mathrm{G}=$ Látex; $\mathrm{H}=$ Pseudofruto; I= Raiz; $\mathrm{J}=$ Semente), Forma de uso ( $\mathrm{a}=$ In natura; $\mathrm{b}=$ Suco; c= Lambedor; d= Compressa; e= Decocção; f= Maceração; g= Infusão; h= Óleo obtido por prensagem). Concordância quanto aos usos principais (CUPc).

\begin{tabular}{|c|c|c|c|c|c|c|}
\hline Táxons & Etnoespécie & Origem & $\begin{array}{c}\text { Parte } \\
\text { utilizada }\end{array}$ & Indicação terapêutica & Forma de uso & CUPc \\
\hline \multicolumn{7}{|l|}{ AMARANTHACEAE } \\
\hline Chenopodium ambrosioides L. & Mastruz & $E$ & $C, D, E$ & $\begin{array}{l}\text { Dor nos ossos; dor nos peitos; } \\
\text { "Colar ossos"; gripe; inflamação; } \\
\text { verme; dor no estômago; úlcera. }\end{array}$ & $a, b, c, d, e$ & 23,0 \\
\hline \multicolumn{7}{|l|}{ AMARYLLIDACEAE } \\
\hline Allium cepa L. & $\begin{array}{l}\text { Cebola de } \\
\text { cabeça }\end{array}$ & E & A & Gripe. & $f$ & \multirow{2}{*}{$\begin{array}{l}7,7 \\
7,7\end{array}$} \\
\hline Allium sativum $\mathrm{L}$. & Alho & E & A & Crise de garganta. & $\mathrm{c}, \mathrm{e}$ & \\
\hline \multicolumn{7}{|l|}{ ANACARDIACEAE } \\
\hline Anacardium occidentale L. & Cajueiro & $\mathrm{N}$ & $\mathrm{B}, \mathrm{H}$ & Inflamação; ferimento; gripe. & $b, e$ & \multirow[t]{2}{*}{15,5} \\
\hline $\begin{array}{l}\text { Myracrodruon urundeuva } \\
\text { Allemão }\end{array}$ & Aroeira & $\mathrm{N}$ & B & $\begin{array}{c}\text { Ferimento; inflamação; bronquite; } \\
\text { cicatrizante. }\end{array}$ & $a, c, e$ & \\
\hline \multicolumn{7}{|l|}{ APOCYNACEAE } \\
\hline Aspidosperma pyrifolium Mart. & Pereiro & $\mathrm{N}$ & B & $\begin{array}{l}\text { Piolho em animais; piolho em } \\
\text { caprinos. }\end{array}$ & e & 7,4 \\
\hline $\begin{array}{l}\text { Calotropis procera (Aiton) } \\
\text { W.T. Aiton }\end{array}$ & Rosa cera & $E$ & G & Dor de dente; verruga. & a & 7,7 \\
\hline Catharanthus roseus (L.) Don. & $\begin{array}{l}\text { Boa noite, bom } \\
\text { dia }\end{array}$ & $E$ & $\mathrm{D}$ & $\begin{array}{l}\text { Ferida na boca; garganta } \\
\text { inflamada. }\end{array}$ & g & 7,7 \\
\hline \multicolumn{7}{|l|}{ ARECACEAE } \\
\hline Cocos nucifera L. & Coqueiro & $E$ & $\mathrm{~F}, \mathrm{~J}$ & $\begin{array}{l}\text { Dor de barriga; vômito; diarreia; } \\
\text { gripe; falta de apetite; tosse; } \\
\text { garganta; privação; dor de ouvido; } \\
\text { fraqueza; dor no estômago; } \\
\text { dor na garganta; febre; "tiriça"; } \\
\text { inflamação intestinal; dor de } \\
\text { cabeça; disenteria. }\end{array}$ & $\mathrm{a}, \mathrm{e}, \mathrm{h}$ & 61,5 \\
\hline \multicolumn{7}{|l|}{ ASPHODELACEAE } \\
\hline Aloe vera (L.) Burm. F. & Babosa & $E$ & $E$ & $\begin{array}{l}\text { Retirar sinal; cicatrizante; } \\
\text { Inflamação; hemorroida; } \\
\text { ferimentos; piolhos; câncer; } \\
\text { estimulante; anti-inflamatório. }\end{array}$ & $\mathrm{a}, \mathrm{c}$ & 23,7 \\
\hline \multicolumn{7}{|l|}{ BORAGINACEAE } \\
\hline Heliotropium indicum (L.) DC & Fedegoso & $\mathrm{N}$ & C, D, E & Inflamação. & $\mathrm{c}, \mathrm{e}$ & 7,7 \\
\hline \multicolumn{7}{|l|}{ BRASSICACEAE } \\
\hline Nasturtium officinale R. Br. & Agrião & $E$ & $\mathrm{D}, \mathrm{E}$ & Garganta inflamada; rouquidão. & $\mathrm{a}, \mathrm{g}$ & 7,7 \\
\hline \multicolumn{7}{|l|}{ BROMELIACEAE } \\
\hline Ananas comosus (L.) Merr. & Abacaxi & $\mathrm{N}$ & $\mathrm{F}$ & Tosse; gripe mal curada. & f & 7,7 \\
\hline \multicolumn{7}{|l|}{ CARICACEAE } \\
\hline Carica papaya L. & $\begin{array}{l}\text { Mamoeiro, } \\
\text { mamão }\end{array}$ & $\mathrm{N}$ & $\mathrm{D}, \mathrm{F}, \mathrm{G}, \mathrm{J}$ & $\begin{array}{l}\text { Comida que faz mal; gripe; } \\
\text { privação; verme; bronquite; "prisão } \\
\text { de ventre"; intestino privado. }\end{array}$ & $\mathrm{a}, \mathrm{c}, \mathrm{e}$ & 15,4 \\
\hline \multicolumn{7}{|l|}{ COMBRETACEAE } \\
\hline Combretum leprosum Mart. & Mofumbo & $\mathrm{N}$ & $\mathrm{B}, \mathrm{F}, \mathrm{I}$ & $\begin{array}{l}\text { Dor de apendicite; pneumonia; } \\
\text { tosse. }\end{array}$ & $\mathrm{a}, \mathrm{c}, \mathrm{e}$ & 15,4 \\
\hline \multicolumn{7}{|l|}{ CONVOLVULACEAE } \\
\hline $\begin{array}{l}\text { Ipomoea asarifolia (Desr.) } \\
\text { Roem. \& Schult. }\end{array}$ & Salsa & E & C, D, E & Lepra; coceira; ferida. & e & 7,7 \\
\hline
\end{tabular}


TABELA 1. Espécies presentes em espaços domésticos citadas para fins medicinais por informantes de São

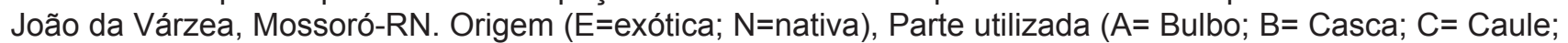
$\mathrm{D}=$ Flor; $\mathrm{E}=$ Folha; $\mathrm{F}=$ Fruto; $\mathrm{G}=$ Látex; $\mathrm{H}=$ Pseudofruto; I= Raiz; $\mathrm{J}=$ Semente), Forma de uso ( $\mathrm{a}=$ In natura; $\mathrm{b}=$ Suco; c= Lambedor; d= Compressa; e= Decocção; f= Maceração; g= Infusão; h= Óleo obtido por prensagem). Concordância quanto aos usos principais (CUPc).

continuação...

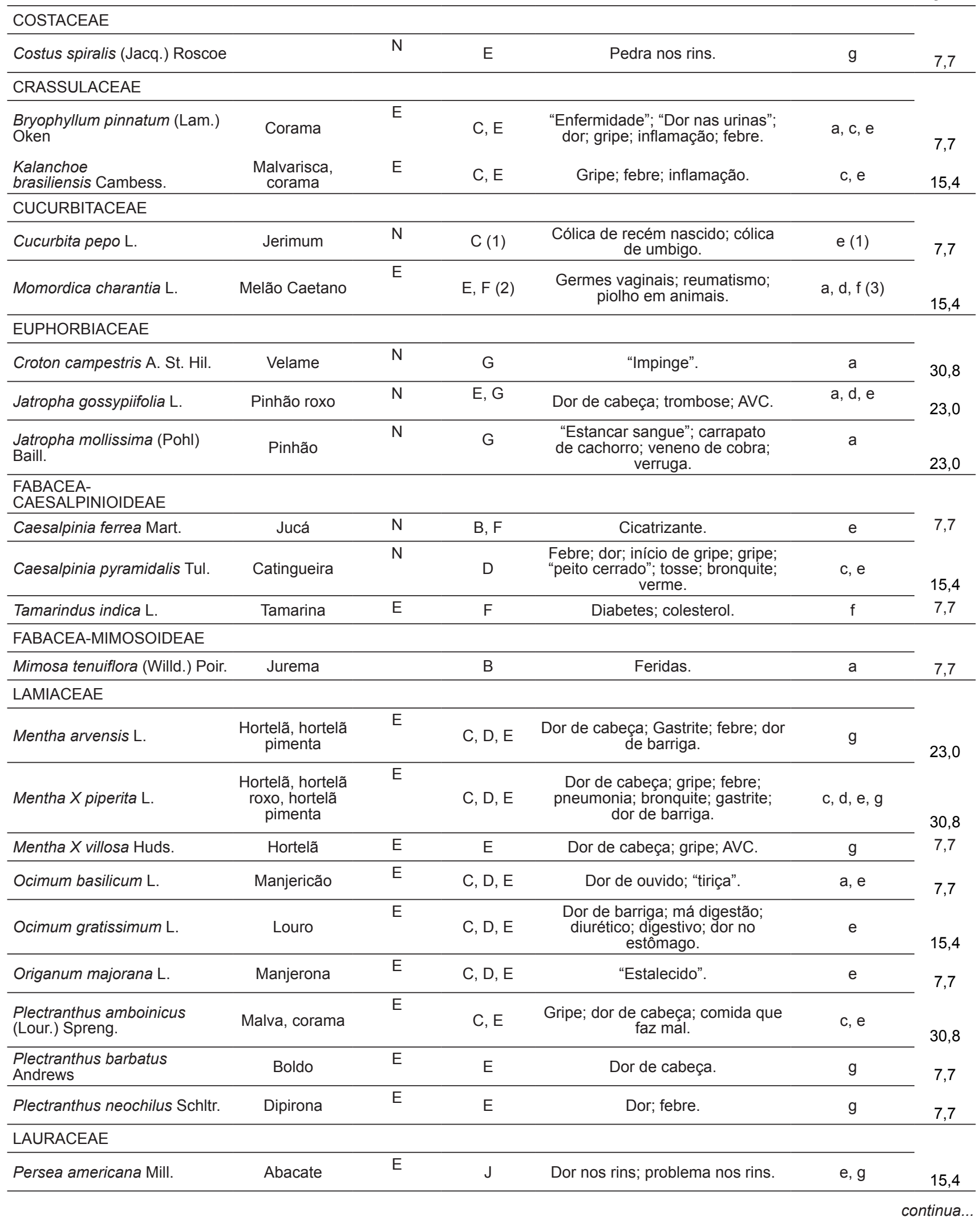

Rev. Bras. PI. Med., Campinas, v.17, n.4, supl. II, p.845-856, 2015. 
TABELA 1. Espécies presentes em espaços domésticos citadas para fins medicinais por informantes de São João da Várzea, Mossoró-RN. Origem ( $E=$ exótica; $N=$ nativa), Parte utilizada ( $A=B$ Bulbo; $B=C a s c a ; C=C a u l e$; $\mathrm{D}=$ Flor; $\mathrm{E}=$ Folha; $\mathrm{F}=$ Fruto; $\mathrm{G}=$ Látex; $\mathrm{H}=$ Pseudofruto; I= Raiz; J= Semente), Forma de uso ( $\mathrm{a}=$ In natura; $\mathrm{b}=$ Suco; c= Lambedor; d= Compressa; e= Decocção; $f=$ Maceração; $g=$ Infusão; $h=$ Óleo obtido por prensagem). Concordância quanto aos usos principais (CUPc).

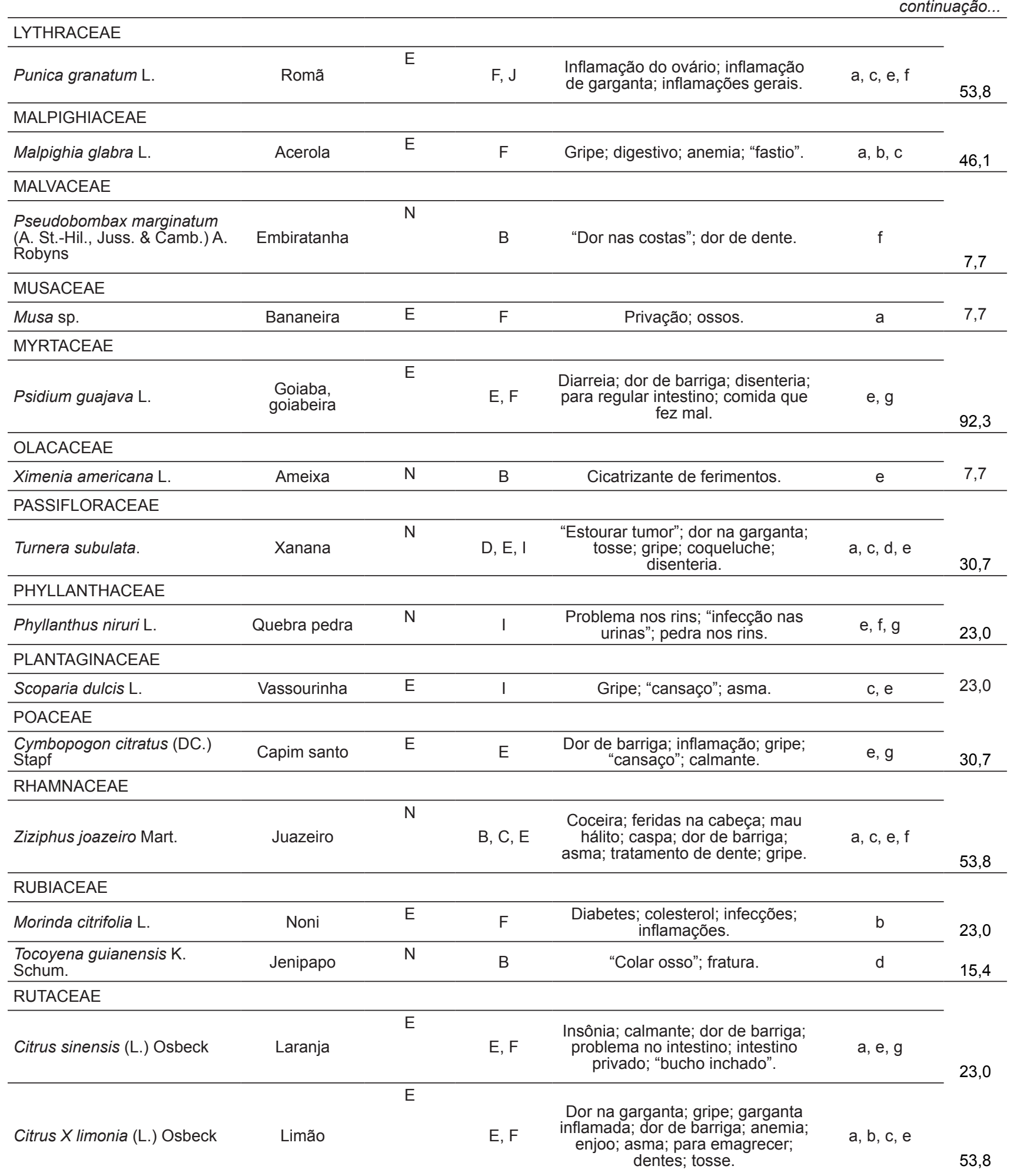


TABELA 1. Espécies presentes em espaços domésticos citadas para fins medicinais por informantes de São

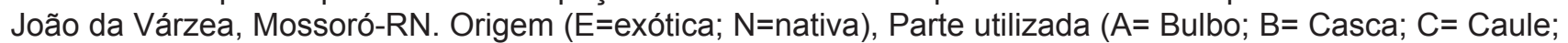
$\mathrm{D}=$ Flor; $\mathrm{E}=$ Folha; $\mathrm{F}=$ Fruto; $\mathrm{G}=$ Látex; $\mathrm{H}=$ Pseudofruto; I= Raiz; $\mathrm{J}=$ Semente), Forma de uso ( $\mathrm{a}=$ In natura; $\mathrm{b}=$ Suco; c= Lambedor; d= Compressa; e= Decocção; f= Maceração; g= Infusão; h= Óleo obtido por prensagem). Concordância quanto aos usos principais (CUPc).

continuação...

\begin{tabular}{|c|c|c|c|c|c|c|}
\hline SAPOTACEAE & & & & & & \\
\hline $\begin{array}{l}\text { Sideroxylon obtusifolium } \\
\text { (Roem. \& Schult.) T. D. Penn. }\end{array}$ & Quixabeira & $\mathrm{N}$ & B & $\begin{array}{l}\text { Inflamação; pancada; ferida; } \\
\text { bronquite; inflamação de útero. }\end{array}$ & $f$ & 15,4 \\
\hline SOLANACEAE & & & & & & \\
\hline Solanum melongena L. & Berinjela & $E$ & $\mathrm{~F}$ & Colesterol alto. & $\mathrm{b}$ & 7,7 \\
\hline VERBENACEAE & & & & & & \\
\hline $\begin{array}{l}\text { Lippia alba (Mill.) N. E. Br. ex } \\
\text { Britton \& P. Wilson }\end{array}$ & $\begin{array}{l}\text { Cidreira, erva } \\
\text { cidreira }\end{array}$ & $\mathrm{N}$ & $C, D, E$ & $\begin{array}{l}\text { Dor de barriga; "moleza no corpo"; } \\
\text { má digestão; " "ressão alta"; } \\
\text { calmante; vômito; insônia. }\end{array}$ & $e, g$ & 7,7 \\
\hline ZINGIBERACEAE & & & & & & \\
\hline $\begin{array}{l}\text { Alpinia zerumbet (Pers.) B. L. } \\
\text { Burtt \& R. M. Sm. }\end{array}$ & $\begin{array}{l}\text { Coluna, } \\
\text { colônia }\end{array}$ & $E$ & E & "Dor na coluna". & g & 7,7 \\
\hline
\end{tabular}

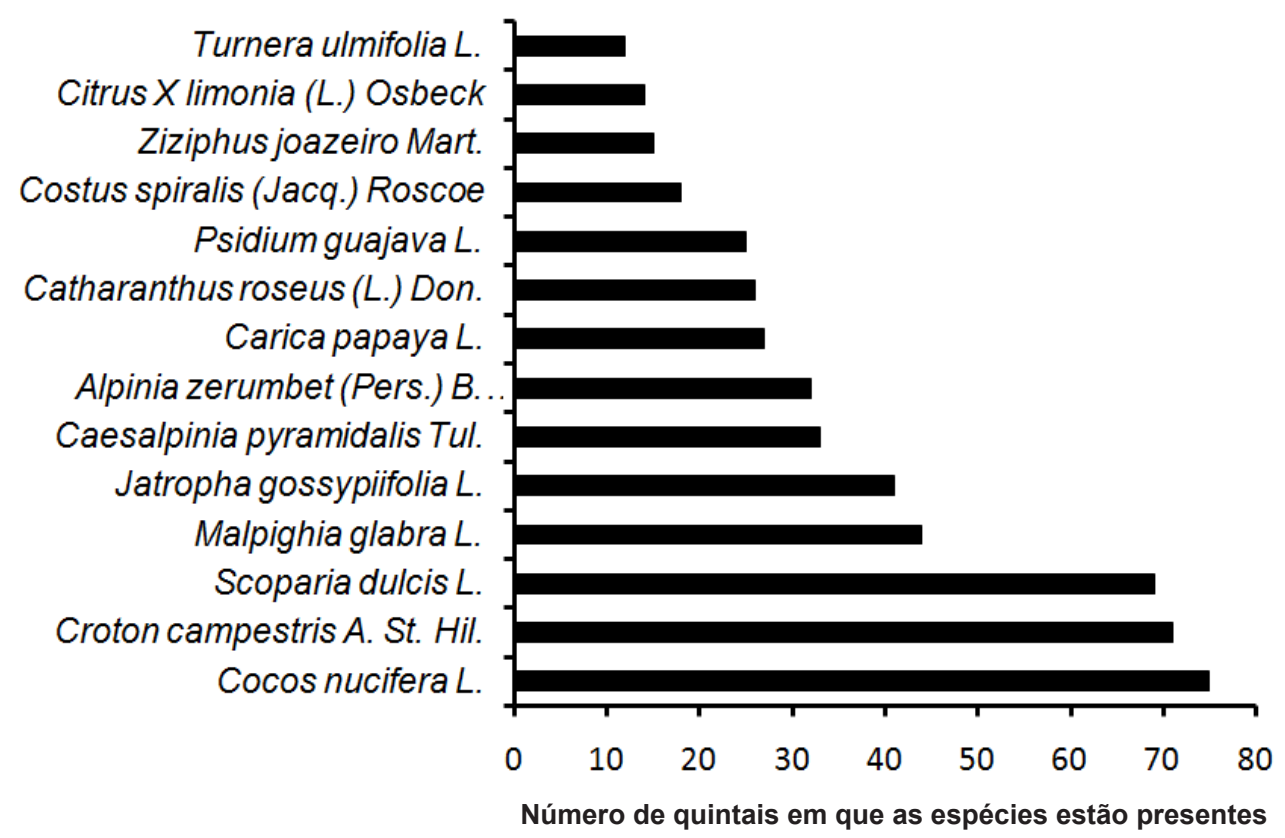

FIGURA 2. Número de indivíduos presentes nos quintais da Comunidade de São João da Várzea, Mossoró, RN. 2012

capim cidreira (Cymbopogom spp.) as espécies mais comuns. Estas diferenças podem ser atribuídas às características da comunidade e do ambiente no nordeste, pois as espécies nativas mantidas nos quintais são de caatinga e o coqueiro é uma das espécies cultivadas preferidas pelos nordestinos em suas residências.

A presença de aproximadamente $38 \%$ de espécies nativas nos quintais evidencia a importância desses espaços para a conservação da biodiversidade. Os estudos realizados em áreas de Caatinga sugerem que esses agroecossistemas podem contribuir para a conservação de plantas nativas e elaboração de estratégias de uso sustentável dos recursos naturais (Albuquerque \& Andrade, 2002; Albuquerque et. al., 2005). Conforme Amaral \& Guarim Neto (2008), os quintais são uma das formas mais antigas de manejo da terra, fato que por si só indica sua sustentabilidade.

A maioria das espécies $(71,9 \%)$ possui mais de uma indicação terapêutica, destacando-se o coqueiro (Cocos nucifera L.), que teve 40 citações 
de indicação terapêutica, tendo seu uso associado ao tratamento de 15 afecções. Os 12 informantes que citaram o coqueiro como medicinal associaram a essa planta outros usos, como alimentação (100\%), artesanato (34\%), sombra (25\%), tecnológico $(25 \%)$ e ornamental (17\%).

Para a utilização como medicinal, foram citadas três formas de preparo e uso, com destaque para o consumo in natura (água de coco) e na forma de óleo, ambas com 7 indicações. O uso através da decocção foi relatado apenas por um dos informantes. Em várias oportunidades, os informantes atribuíram à água de coco a propriedade de soro, não sendo relatada contraindicação da mesma.

Em outros estudos etnobotânicos, o coqueiro também foi considerado espécie medicinal. Em São Miguel-RN, seu consumo in natura foi indicado para dor de barriga, vômito, gripe e diarreia (Freitas et al., 2012). As mesmas indicações foram observadas por Morais (2011) em Caraúbas-RN. Em Apodi-RN, Paulino et al. (2012) verificaram que o óleo do coco é utilizado como cicatrizante. Carneiro (2009) em uma revisão a respeito das espécies de uso medicinal na Região Centro Oeste do Brasil, verificou que o coqueiro foi citado por vários autores para tratamento de tuberculose, hemorroidas, rins, infecção urinária, úlcera, infecção nos rins, verme, disenteria, anemia e derrame, seja utilizando-se o fruto, ou a flor.

O modo de administração que mais se destacou foi por via oral, sobretudo na forma de decocção que foi citada como alternativa ou única forma de preparo para 33 espécies medicinais $(58 \%)$. Resultados semelhantes foram encontrados por Amorozo (2002), em que a administração via oral sob a forma de chá foi verificada para 124 espécies coletadas de ambientes diversos em Santo Antônio do Leverger-MT. A decocção também foi citada como a forma de preparo mais utilizada em outros estudos (Silva \& Sablayrolles, 2009; Siviero et al., 2012).

Outras formas de preparo foram citadas para algumas espécies, como consumo in natura, suco, lambedor, compressa, maceração, infusão e óleo. O lambedor é uma preparação espessada com açúcar, sendo geralmente feita a partir de plantas usadas para problemas respiratórios, como tosse e bronquite (Lorenzi \& Matos, 2008).

Dez partes vegetais foram mencionadas para uso medicinal. Do total de preparações caseiras registradas, as folhas foram empregadas em $32,6 \%$ delas, seguidas dos frutos (20\%), cascas $(10,2 \%)$ e flores $(10,2 \%)$. A maioria dos autores que trabalha com etnobotânica de plantas medicinais também destaca a folha como a parte vegetal mais utilizada nessas preparações (Mosca \& Loiola, 2009; Silva \& Sablayrolles, 2009; Albertasse et al., 2010; Guerra et al., 2010; Freitas et al., 2012).

Este significativo uso de folhas deve-se, provavelmente, à frequente utilização de plantas medicinais herbáceas cultivadas em espaços domésticos pelas pessoas da comunidade, cujas folhas estão disponíveis durante o ano todo. Outros estudos alegam que a maioria das espécies vegetais tende a concentrar os princípios ativos nas folhas, fornecendo indicativo importante na estratégia de defesa contra herbivoria (Stepp, 2001), o que corrobora a importância do uso destas partes vegetais de plantas medicinais.

Os informantes citaram um total de 67 agravos que podem ser tratados com espécies medicinais. O maior número de plantas medicinais foi indicado para o tratamento de gripe (18 spp.), disenteria (11 spp.), inflamações gerais (11 spp.) e como cicatrizante de ferimentos (10 spp.). A gripe e a disenteria também se destacaram como as indicações terapêuticas mais citadas durante as entrevistas, com 42 e 30 citações, respectivamente. Resultados semelhantes foram observados no uso de plantas medicinais por colonos migrantes nordestinos em Santarém - PA (Silva \& Sablayrolles, 2009).

Em estudo sobre o uso popular de plantas medicinais no Rio Grande do Norte várias espécies de plantas foram citadas para os mesmos grupos de doenças, sendo que o tratamento de gripe/ garganta/ouvido foi o mais citado (Mosca \& Loiola, 2009). Maior número de espécies foi citado para o tratamento da gripe, entre os moradores entrevistados em Mangaratiba-RJ (Medeiros et al., 2004). Possivelmente, isso se deve à maior frequência desse tipo de agravo e à crença generalizada de que é algo que não precisa de médico.

Das 57 espécies citadas como medicinal apenas $10(17,6 \%)$ tiveram alguma contraindicação expressa pelos informantes. Entre as espécies que não tiveram contraindicações relatadas estão o pinhão (Jatropha mollissima (Pohl) Baill.) e o pinhão roxo (Jatropha gossypiifolia L.), que são altamente tóxicas e que tiveram a ingestão do látex como indicação de uso. O consumo do látex de pinhão (Jatropha mollissima (Pohl) Baill.) também foi relatado por Roque et al. (2010) em uma comunidade rural no município de Caicó-RN. Lorenzi \& Matos (2008) relatam a ocorrência de substâncias com propriedades tóxicas no pinhão roxo (Jatropha gossypiifolia L.), e afirmam que a administração oral deve ser desaconselhada.

As espécies que apresentaram CUPc maior que $50 \%$ foram Ziziphus joazeiro Mart. $(53,8)$, Citrus $X$ limonia (L.) Osbeck $(53,8)$, Punica granatum L. $(53,8)$, Cocos nucifera L. $(61,5)$ e Psidium guajava L. $(92,3)$. O índice CUPc é referido à fidelidade

Rev. Bras. PI. Med., Campinas, v.17, n.4, supl. II, p.845-856, 2015. 
quanto a principal utilização da espécie citada pelos informantes, podendo sugerir que as espécies com um índice de concordância relativamente alto podem ser promissoras para realização de estudos farmacológicos que possam corroborar a eficácia de suas propriedades terapêuticas (Amorozo \& Gély, 1988). Assim estas espécies merecem maiores estudos farmacológicos, principalmente Psidium guajava que é cultivada em todo o Brasil como frutífera, e usada pela população para diarreia.

Os valores percentuais de Concordância corrigida quanto aos Usos Principais para cada uma das espécies (CUPc) foram utilizados em outros levantamentos etnobotânicos realizados no Brasil. As espécies que também foram encontradas nestes trabalhos com o índice de CUPc maior que $50 \%$ foram Eugenia uniflora (76\%), Achyrocline satureioides (68\%) e Psidium guajava (64\%) por Vendruscolo \& Mentz (2006), Plectranthus barbatus (93\%), P. neochilus (71,8\%), Hyptis suaveolens $(64,5 \%)$ e Apium graveolens $(63,9 \%)$ por Meyer et al., (2012), Sambucus nigra (76,9\%), Croton antisiphiliticus (61,5\%), Mentha pulegium (64\%) e Plectranthus barbatus (100\%) por Alves \& Povh (2013).

Segundo Vendruscolo \& Mentz (2006), através do CUPc são apontados os usos mais difundidos e aceitos pela população para uma espécie. A aceitação de um uso por uma população implica em maior segurança quanto à relativa eficácia do uso proposto, pois ele é provavelmente utilizado ou conhecido por muitos informantes.

Quanto à diversidade de espécies medicinais encontradas nos espaços domésticos estudados, o Índice de diversidade de ShannonWiener $\left(H^{\prime}\right)$ na base 10 foi de 1,62 décits/indivíduo e na base e, 3,73 nats/indivíduos. Esses valores são considerados baixos, se comparados aos de outras pesquisas etnobotânicas, como Paulino et al. (2012) que estudando as plantas medicinais da caatinga e de quintais utilizadas no Sitio do Góis, Apodi$\mathrm{RN}$, encontraram valores de $\mathrm{H}^{\prime}=2,59$ e Cunha \& Bortolotto (2011), em trabalho sobre a etnobotânica de plantas medicinais no Assentamento Monjolinho em Anastácio-MS, verificaram uma alta diversidade de usos das espécies, com $\mathrm{H}^{\prime}=5,03$, refletindo a disponibilidade de recursos vegetais nativos $\mathrm{e}$ cultivados e a familiaridade da população com estes recursos.

A baixa diversidade encontrada no presente estudo deve-se, possivelmente, ao fato do trabalho abordar apenas as plantas medicinais presentes nos quintais, enquanto os demais trabalhos citados abrangeram plantas medicinais encontradas em outros ambientes, sobretudo em áreas de vegetação nativa, que são alvo de extrativismo.

\section{CONCLUSÕES}

Os quintais são sistemas que atuam como uma estratégia de uso mais sustentável dos recursos medicinais por conservarem também as espécies nativas. As espécies medicinais mais frequentes nos quintais são arvores frutífero-alimentícias importantes para a renda familiar. A gripe e desinteria são os principais agravos de saúde tratados com espécies medicinais pela comunidade. Ziziphus joazeiro Mart., Citrus X limonia (L.) Osbeck, Punica granatum L., Cocos nucifera L. e Psidium guajava L. são espécies que merecem maiores estudos farmacológicos.

\section{REFERÊNCIAS}

ABDEL-MOGIB, M. et al. Chemistry of the Genus Plectranthus. Molecules, v.7, p. 271-301, 2002.

AGUIAR, L.C.G.G.; BARROS, R.F.M. Plantas medicinais cultivadas em quintais de comunidades rurais no domínio do cerrado piauiense (Município de Demerval Lobão, Piauí, Brasil). Revista Brasileira de Plantas Medicinais, v.14, n.3, p. 419-434, 2012.

ALBERTASSE, P.D. et al. Plantas medicinais e seus usos na comunidade da Barra do Jucu, Vila Velha, ES. Revista Brasileira de Plantas Medicinais, v.12, n.3, p. 250-260, 2010.

ALBUQUERQUE, U. P.; ANDRADE, L. H. C. Uso de recursos vegetais da caatinga: o caso do agreste do estado de Pernambuco (Nordeste do Brasil). Interciência, v. 27, n. 7, p. 336-346, 2002.

ALBUQUERQUE, U.P. et al. Structure and Floristics of Homegardens in Northeastern Brazil. Journal of Arid Enviroments, v.62, n.3, p. 491-506. 2005.

ALBUQUERQUE, U.P.; LUCENA, R.F.P.; ALENCAR, N.L. Métodos e técnicas para coleta de dados etnobiológicos. In: ALBUQUERQUE, U.P.; LUCENA, R.F.P.; CUNHA, L.V.F.C. Métodos e técnicas na pesquisa etnobiológica e etnoecológica. $3^{a}$ ed. Recife: NUPEEA, 2010. p.39-64.

ALVES, M.; ARAÜJO, M.F.L.; GUSMÃO, C.L.S.; LIRA NETO, A.C.; CARVALHO, R.; BENKO-ISSEPON, A.M. Diversity and uses of genus Croton (Euphorbiaceae) in northeastern of Brazil. In: RAI, M.; CORDELL, G.A.; MARTINEZ, J.L.; MARINOFF, M.; RASTRELLI, L. (Eds.) Medicinal plants: biodiversity and drugs. $1^{\text {a }} \mathrm{ed}$. Londres: CRC Press, 2012. p.106-141.

ALVES, G.S.P.; POVH, J.A. Estudo etnobotânico de plantas medicinais na comunidade de Santa Rita, Ituiutaba - MG. Biotemas, v.26, n.3, p. 231-242, 2013.

AMARAL, C.N.; GUARIM-NETO, G. Os quintais como espaços de conservação e cultivo de alimentos: um estudo na cidade de Rosário Oeste (Mato Grosso, Brasil). Boletim do Museu Paraense Emílio Goeldi, v. 3, n. 3, p. 329-341, 2008.

AMOROZO, M. C. M.; VIERTLER, R. B. A abordagem qualitativa na coleta e análise de dados em etnobiologia e etnoecologia. In: ALBUQUERQUE, U. P.; LUCENA, R. F. P.; CUNHA, L. V. F. C. Métodos e técnicas na pesquisa etnobiológica e etnoecológica. $3^{a}$ ed.

Rev. Bras. PI. Med., Campinas, v.17, n.4, supl. II, p.845-856, 2015. 
Recife: NUPEEA, 2010. p. 67-82.

AMOROZO, M.C.M. Uso e diversidade de plantas medicinais em Santo Antônio do Leverger, MT, Brasil. Acta Botânica Brasílica, v.16, n.2, p.189-203, 2002.

AMOROZO, M.C.M.; GÉLY, A. Uso de plantas medicinais pelos caboclos do baixo Amazonas. Barcarena, PA, Brasil. Boletim do Museu Paraense Emílio Goeldi, v.4, n.1, p. 47-131, 1988.

ANGEL-PEREZ, A.L.D.; MARTIN, A.M.B. Totonac homegardens and natural resources in Veracruz, Mexico. Agriculture and Human Values, v.21, p.329346, 2004.

APG. An date of the Angiosperm Phylogeny Group classification for the orders and families of flowering plants: APG II. Botanical Journal of the Linnean Society, v.141, n.1, p. 399-436, 2003.

AZEVEDO, C.M.A.A regulamentação do acesso aos recursos genéticos e aos conhecimentos tradicionais associados no Brasil. Biota Neotropica, v.5, p.19-27, 2005.

BAILEY, K. Methods of Social Research. 4. ed. New York: The Free Press, 1994. 588p.

BEGOSSI, A. Use of ecological methods in ethnobotany: diversy indices. Economic Botany, v.50, n.3, p. 280289, 1996.

CARNEIRO, M.R.B. A flora medicinal no Centro Oeste do Brasil: um estudo de caso com abordagem etnobotânica em Campo Limpo de Goiás. 2009. 242f. Dissertação (Mestrado em Sociedade, Tecnologia e Meio Ambiente) - Centro Universitário de Anápolis, Unievangélica, Anápolis.

CARNIELLO, M. A. et al. Quintais urbanos de Mirassol D'Oeste-MT, Brasil: uma abordagem etnobotânica. Acta Amazônica, v.40, n. 3, p. 451- 470, 2010.

COSTANTIN, A.M. Quintais Agroflorestais na visão dos agricultores de Imaruí - SC. 2005. 120f. Dissertação (Mestrado em Agroecossistemas) - Centro de Ciências Agrárias. Universidade Federal de Santa Catarina, Florianópolis.

CUNHA, S.A.; BORTOLOTTO, I.M. Etnobotânica de Plantas Medicinais no Assentamento Monjolinho, município de Anastácio, Mato Grosso do Sul, Brasil. Acta Botânica Brasílica, v.25, n.3, p. 685-698, 2011.

DELWING, A.B. et al. A etnobotânica como ferramenta da validação do conhecimento tradicional: manutenção e resgate dos recursos genéticos. Revista Brasileira de Agroecologia, v.2, n.1, p. 421-425, 2007.

DIEGUES, A.C.S. O mito moderno da natureza intocada. 4. ed. São Paulo: HUCITEC, 2008. 169p.

EMATER. Instituto de Assistência Técnica e Extensão Rural do Rio Grande do Norte. Diagnóstico das comunidades do Polo Passagem do Rio, MossoróRN. Mossoró-RN: EMATER-RN, 2012. 54p.

FLORENTINO, A.T.N. et al. Contribuição de quintais agroflorestais na conservação de plantas da Caatinga, município de Caruaru, PE, Brasil. Acta Botânica Brasílica, v.21, n.1, p. 37-47, 2007.

FREITAS, A.V.L.; et al. Plantas medicinais: um estudo etnobotânico nos quintais do Sítio Cruz, São Miguel, Rio Grande do Norte, Brasil. Revista Brasileira de Biociências, v.10, n.1, p. 48-59, 2012

FREITAS, A.V.L. Recursos genéticos em quintais e comercialização de plantas de uso medicinal no município de São Miguel- RN. 2009. 192p. Dissertação (Mestrado em Agronomia: Fitotecnia) - Programa de Pós-Graduação em Agronomia, UFERSA. Mossoró, RN.

GUARIM NETO, G.; AMARAL, C.N. Aspectos etnobotânicos de quintais tradicionais dos moradores de Rosário Oeste, Mato Grosso, Brasil. Polibotánica, n.29, p.191-212, 2010.

GUERRA, A.M.N.M. et al. Utilização de plantas medicinais pela comunidade rural Moacir Lucena, Apodi-RN. Bioscience Journal, v. 26, n. 3, p. 442-450, 2010.

GUERRA, A.M.N.M. et al. Plantas medicinais e hortaliças usadas para cura de doenças em residências da cidade de Mossoró-RN. Revista Verde, v.2, n.1, p. 70-77, 2007.

IDEMA. Instituto de Desenvolvimento Sustentável e Meio Ambiente do Rio Grande do Norte. Perfil do seu município Mossoró. v.10, p.7-8, 2008, Natal, RN. Disponível em: http://adcon.rn.gov.br/ACERVO/idema/ DOC/DOC000000000013950.PDF, Acesso em: 12 out. 2014.

KUMAR B.M.; NAIR P.K.R. The enigma of tropical homegardens. Agroforest Systems, v.61, p.135-152, 2004.

LEONEL, J.C. Quintais para Vida: tecnologia social no semiárido. $1^{\text {a }}$. ed. Fortaleza: CETRA, 2010. 130p.

LIPORACCI, H.S.N; SIMÃO, D.G. Levantamento etnobotânico de plantas medicinais nos quintais do Bairro Novo Horizonte, Ituiutaba, MG. Revista Brasileira Plantas Medicinais, v.15, n.4, p. 529-540, 2013.

LORENZI, H.; MATOS, F.J.A. Plantas medicinais no Brasil: nativas e exóticas cultivadas. Nova Odessa: Instituto Plantarum, 2008. 512p.

MEDEIROS, M.F.T. et al. Plantas medicinais e seus usos pelos sitiantes da Reserva Rio das Pedras, Mangaratiba, RJ, Brasil. Acta Botanica Brasílica, v. 8, n.2, p. 391399, 2004.

MEYER, L . et al. Etnobotânica na comunidade de Santa Bárbara, Ascurra, Santa Catarina, Brasil. Revista Brasileira de Biociências, v.10, n.3, p. 258-266,. 2012

MOBOT. Missouri Botanical Garden. Tropicos. Disponível em: <http://www.tropicos.org/theplantlist/>. Acesso em: 08 mar. 2014.

MORAIS, V.M. Etnobotânica nos quintais da comunidade de Abderramant em Caraúbas-RN. 2011. 112f. Tese (Doutorado em Fitotecnia: Área de concentração em Agricultura Tropical) - Programa de Pós-Graduação em Fitotecnia, Universidade Federal Rural do Semiárido, Mossoró.

MOSCA, V.P.; LOIOLA, M.I.B. Uso popular de plantas medicinais no Rio Grande do Norte, nordeste do Brasil. Revista Caatinga, v.22, p.225-234, 2009.

MOURA, C.L.; ANDRADE, L.H.A. Etnobotânica em quintais urbanos nordestinos: um estudo no bairro da Muribeca, Jaboatão dos Guararapes, PE. Revista Brasileira de Biociências, v.5, n.1, p. 219-221. 2007.

PASA, M.C. et al. Enfoque etnobotânico das categorias de uso das plantas na unidade de paisagem quintal, comunidade Fazenda Verde em Rondonópolis, MT. Biodiversidade, v.7, n.1, p. 3-13, 2008.

PASA, M.C. et al. Estudo etnobotanico na comunidade de Conceicao-Acu (Alto da Bacia do Rio Aricá Açu, MT, Brasil). Acta botânica brasileira, v.19, n.2, p. 195-207, 2005. 
PAULINO, R.C. et al. Medicinal plants at the Sítio do Gois, Apodi, Rio Grande do Norte State, Brazil. Revista Brasileira de Farmacognosia, v.22, n.1, p. 29-39, 2012.

RONDON NETO, R.M. et al. Os quintais agroflorestais do assentamento rural rio da areia, município de Teixeira Soares, PR. Cerne, v.10, n.1, p. 125-135, 2004.

ROQUE, A.A. et al. Uso e diversidade de plantas medicinais da Caatinga na comunidade rural de Laginhas, município de Caicó, Rio Grande do Norte (Nordeste do Brasil). Revista Brasileira de Plantas Medicinais, v.12, n.1, p. 31-42. 2010.

SILVA, E.R.R.; SABLAYROLLES, M.G.P. Quintais agroflorestais por colonos migrantes: as plantas medicinais em Vila Nova, Mojuí dos Campos (Santarém/ PA). Anais... In: VII Congresso Brasileiro de Sistemas Agroflorestais, 2009, Luziânia. Diálogo e integração de saberes em sistemas agroflorestais para sociedades sustentáveis, 2009. p.1-4.

SILVA, T.S.; FREIRE, E.M.X. Abordagem etnobotânica sobre plantas medicinais citadas por populações do entorno de uma unidade de conservação da caatinga do Rio Grande do Norte, Brasil. Revista Brasileira de Plantas Medicinais, v.12, n.4, p. 427-435, 2010.

SIVIERO, A. et al. Plantas medicinais em quintais urbanos de Rio Branco, Acre. Revista Brasileira de Plantas Medicinais, v.14, n.4, p. 598-610, 2012.

STEPP, J.R.; MOERMAN, D.E. The importance of weeds in ethnopharmacology. Journal of Ethnopharmacology, v.75, p.19-23, 2001.

VENDRUSCOLO, G.S.; MENTZ, L.A. Estudo da concordância das citações de uso e importância das espécies e famílias utilizadas como medicinais pela comunidade do bairro Ponta Grossa, Porto Alegre, RS, Brasil. Acta Botânica Brasílica, v. 20, n.2, p. 367-382, 2006.

VISBISKI, V. N. Sistemas silvipastoris em Imaruí SC: a construção de um sonho comum. 2003. $141 \mathrm{f}$. Dissertação (Mestrado em Agrossistemas) - Centro de Ciências Agrárias. Universidade Federal de Santa Catarina, Florianópolis, 2003.

WEZEL, A.; BENDER, S. Plant species diversity of homegardens of Cuba and its significance for household food supply. Agroforestry Systems, v.57, p.39-49, 2003. 